\title{
Originales
}

\section{Prevalencia de lactancia materna en menores de 6 meses en el departamento del Huila, Colombia}

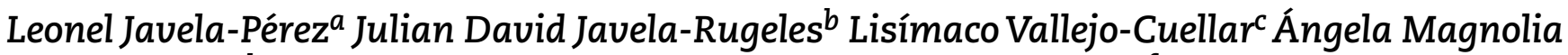 Rios-Gallardo ${ }^{d}$ Pedro León Reyes-Gaspar Edilberto Suaza-Calderónf Claudia Leonor Trujillo- Bahamon $^{g}$ José Domingo Alarcón ${ }^{h}$}

\author{
a Médico, Especialista en Pediatría, Docente de Pediatría Universidad Surcolombiana, email: leoneljavelaperez@yahoo.es \\ b Médico investigador, Universidad Surcolombiana, Neiva (Colombia). julianjavela@hotmail.com \\ c Licenciado en Educación Física, PhD Bioestadistica, Coordinador de Unidad \\ d. Psicóloga, Doctorada en Psicología con orientación en Neurociencia cognitiva aplicada, Vicerrectora de Investigaciónes y Proyección Social USCO, \\ angela.rios@usco.edu.co \\ e Médico, Doctorado en Ciencias de la Salud, Rector USCO, reyesgaspar@gmail.com \\ f Enfermero, Especialista en Epidemiología, Docente USCO, edisuaza@gmail.com \\ g Nutricionista Dietista, Especialista en Salud Familiar Integral, Secretaría de Salud Departamental-Huila, claudia.trujillo@huila.gov.co \\ h Médico, Doctorado en Salud Publica y Metodología de la Investigación Biomédica, Decano Facultad de Salud USCO, jodomala@gmail.com
}

INFORMACIÓN DEL ARTÍCULO

Historia del artículo:

Recibido el 03 de julio de 2018

Aceptado el 20 de septiembre de 2018

Palabras clave:

lactancia materna

estadística

datos numéricos

\section{RESUMEN}

Objetivos: conocer la prevalencia de lactancia materna (exclusiva, predominante y con alimentación complementaria) en bebés $<6$ meses en el Huila y subregiones, así como factores relacionados.

Métodos: estudio con diseño correlacional, descriptivo-transversal, en madres de bebés $<6$ meses realizado entre octubre-diciembre de 2015. Del total de bebés $<6$ meses del Huila ( $\mathrm{N}=$ 9 755), se realizó una encuesta a madres de 335 bebés, incluyendo los 37 municipios, el área rural y urbana. Se realizó muestreo no probabilístico por conveniencia utilizando información del Instituto Colombiano de Bienestar Familiar, centros asistenciales en salud y búsqueda aleatorizada simple para ubicar a las madres.

Resultados: en < 6 meses se encontró prevalencia de lactancia materna del $95.8 \%$; lactancia materna exclusiva obtuvo $58.5 \%$; predominante, $17.6 \%$; y alimentación complementaria a lactancia materna 19.7\%. Categorizando por grupos etarios (meses de 0-1, 2-3 y 4-5), la prevalencia de la lactancia materna exclusiva fue $74.8,56.4$ y $31.3 \%$, respectivamente. Al sumar la lactancia materna exclusiva con la predominante (lactancia materna completa), las prevalencias fueron $85.3,72.2$ y $63.8 \%$, respectivamente.

Conclusiones: se logró conocer por primera vez para el departamento del Huila las prevalencias en lactancia materna. La prevalencia de lactancia materna, lactancia materna exclusiva, predominante y con alimentación complementaria, tanto en total de $<6$ meses, categorizando por subgrupos etarios, es mayor que la media referida para Colombia en su Encuesta Nacional de Situación Nutricional (Ensin-2010). Se logró establecer indicadores para subregiones del Huila y caracterización de factores relacionados con lactancia materna.

*Autor para correspondencia. Leonel Javela Perez Correo electrónico: leoneljavelaperez@yahoo.es

Como Citar: Javela L., Javela J., Vallejo L., Rios A. Reyes P., Suaza E., Trujillo C., Alarcón J. Prevalencia de lactancia materna en menores de 6 meses en el departamento del Huila, Colombia. Pediatr. 2018;51(1): 1-8. 


\title{
Prevalence of Breastfeeding in Children Under 6 Months in the Department of Huila, Colombia.
}

\author{
A B S T R A C T
}

Objectives: Know the prevalence of breastfeeding, breastfeeding exclusively, predominantly with complementary feedings in $<6$ months in Huila and subregions and related factors.

Methods: Study with design correlational, description- transversal, in mothers of $<6$ months carried out between October-December of 2015. Of $9755<6$ months of Huila, survey was carried out to mothers of 335 babies including the 37 municipalities, urban and rural. Sampling was carried out non-probability convenience using information from the Instituto Colombiano de Bienestar Familiar, care centers health and simple random search to locate to the mothers.

\section{Keywords:}

Breast Feeding

statistics

numerical data
Results: In $<6$ months was found prevalence of $95.8 \%$ breastfeeding, breastfeeding exclusive $58,5 \%$, predominantly $17.6 \%$, and complementary breastfeeding $19.7 \%$ feeding. Categorizing by age grind (months 0-1, 2-3, 4-5), 74.8\% exclusive breastfeeding prevalence, 56.4\% and 31.1 $\%$ respectively. Uniting exclusive breastfeeding more predominant prevalences were 85.3 , 72.2 y $63.8 \%$ respectively.

Conclusions: In the Huila, the prevalence of breastfeeding maternal, lactation maternal exclusive, predominantly and with power complementary, both in total of $<6$ months, as by categorizing by subgroups age, is better than the aforementioned for Colombia in its Encuesta Nacional de Situación Nutricional (Ensin-2010). It was possible to establish indicators for subregions of the Huila and characterization of factors related to breastfeeding.

\section{Introducción}

La lactancia materna (LM) es un factor determinante en la salud de una población durante todo su ciclo de vida. Para el niño(a), LM implica recibir el mejor alimento funcional que existe, ser defendido por la madre a través de un sistema de defensas relacionadas con el amamantamiento y recibir estímulos afectivos que fomentan su desarrollo neuro-psico-social (1). La LM fomenta la salud de las madres, pues reduce el riesgo de enfermedades como el cáncer de ovario y mama. Además, la LM disminuye costos en alimentación del núcleo familiar y beneficia al ambiente (2).

La OMS recomienda que los niños sean alimentados en sus primeros 6 meses de vida con lactancia materna exclusiva (LME) y, posteriormente, reciban LM (y alimentación complementaria) por 2 años o más. A pesar de los grandes beneficios, las recomendaciones sobre LM no se alcanzan en la mayoría de países, lo que produce altas tasas de malnutrición, morbi-mortalidad infantil e incremento de enfermedades crónicas no transmisibles en adultos (3).

En las encuestas nacionales de situación nutricional en Colombia, la prevalencia de LM en <6 meses en 2005(4) y 2010(5) fueron de 90.6 y $95 \%$, respectivamente. La prevalencia de LME en $<6$ meses fue 46.8 y $42.9 \%$ respectivamente. En Colombia, la gran diversidad de regiones aporta diferencias en LM, las cuales no son representadas claramente en los indicadores nacionales.

Para el departamento del Huila no se conocen prevalencias en LM. La Ensin reporta para el Huila la mediana de LME; indicador no sugerido por la OMS. Las encuestas nacionales no han pretendido evaluar ampliamente LM en cada departamento y sus subregiones, por lo que se hace evidente la falta información específica..

Dada la gran importancia de la LM, se consideró pertinente realizar en el Huila y subregiones una evaluación de su prácti- ca. Este estudio buscó obtener prevalencias y conocimiento de factores relacionados con la LM entre los huilenses que permitieran la formulación de mejores planes de promoción y apoyo a la LM, así como las consecuentes mejoras de los resultados en la salud de su población.

\section{Métodos}

El estudio se realizó en el Huila, departamento del sur-occidente de Colombia que contiene 37 municipios en 4 subregiones: Norte, Centro, Occidente y Sur; las cuales cuentan con 15, 8, 5 y 9 municipios, respectivamente.

El estudio fue de diseño correlacional y descriptivo-transversal. Se realizó una encuesta a madres de bebés $<6$ meses. La población objeto fue de 9755 niños, que corresponden a la población de $<6$ meses del Huila ${ }^{1}$. Para el tamaño de muestra se consideró prevalencia de LME en $<6$ meses en Colombia (Ensin-2010: $42.9 \%$ ). Se asumió error del 5 \% y nivel de confianza del $95 \%$. Mediante la fórmula para poblaciones finitas se estableció el tamaño de muestra de 362 bebés. La muestra final fue de 335 niños, por pérdida de datos (7.4\%). Se incluyeron $<6$ meses de los 37 municipios del departamento. Se realizó muestreo no probabilístico por conveniencia utilizando información del Instituto Colombiano de Bienestar Familiar (ICBF) y centros asistenciales en salud para ubicar a las madres; cuando no se logró alcanzar la meta prevista, se hizo búsqueda aleatorizada simple. Las madres firmaron consentimiento informado. Se realizó la encuesta, por personal previamente capacitado, entre los meses de octubre y diciembre 2015.

\footnotetext{
1 Información suministrada por la Secretaría de Salud del Huila.
} 
La información se consolidó en una matriz de datos elaborada en el software Excel, versión 2010. El análisis estadístico se realizó a través del software estadístico SPSS, versión 23. Para el análisis univariado se implementó estadística descriptiva, frecuencias relativa y absoluta, medidas de tendencia central y medidas de dispersión. La normalidad de datos se determinó por medio del test de Kolmogorov-Smirnov. En el análisis bivariado se aplicó el test de Chi-cuadrado para variables cualitativas y el Anova de un factor y Levene para identificar varianzas homogéneas en variables cuantitativas; para los no paramétricas se usó el test de Kruskan-Wallis, U de
Mann-Whitney y Moses. El nivel de significancia permitido fue de $\mathrm{p} \leq 0.05$.

Para definición de variables se siguieron las siguientes recomendaciones de la OMS (2007) sobre indicadores de alimentación en lactantes y niños pequeños (ver cuadro siguiente). Se preguntó al cuidador sobre alimentos recibidos por el bebé en 24 horas previas (6).

OMS. Consenso global: indicadores para evaluar la práctica de alimentación en infantes y niños pequeños. Washington, noviembre 2007.

\begin{tabular}{|c|c|c|}
\hline Práctica alimentaria infantil & $\begin{array}{l}\text { Requiere que el niño esté } \\
\text { recibiendo }\end{array}$ & Puede incluir \\
\hline Lactancia materna exclusiva (LME) & Solamente LM & $\begin{array}{l}\text { Rehidratación oral, gotas (vitaminas, } \\
\text { medicinas, minerales) }\end{array}$ \\
\hline $\begin{array}{l}\text { Lactancia materna predominante } \\
\text { (LMP) }\end{array}$ & $\begin{array}{l}\text { LM como fuente principal de } \\
\text { alimentación y líquidos que no sean } \\
\text { otra leche }\end{array}$ & $\begin{array}{l}\text { Líquidos (agua, jugo de fruta, bebidas), } \\
\text { rehidratación oral, gotas (vitaminas, } \\
\text { medicinas, minerales) }\end{array}$ \\
\hline $\begin{array}{l}\text { Alimentación complementaria (a la } \\
\text { LM) }\end{array}$ & LM y alimentos semisólidos y sólidos & \\
\hline $\begin{array}{l}\text { Cualquier alimento, incluyendo leche } \\
\text { no materna }\end{array}$ & & \\
\hline
\end{tabular}

\section{RESULTADOS}

La tabla 1 muestra una distribución equilibrada por género y área de residencia. La mediana de edad fue de 71 días. Por grupos etarios hubo disminución progresiva en el número de bebés conforme aumentó la edad. Por regiones, la población osciló entre 80-88 bebés por cada subregión. En la subregión Norte predominaron los bebes de área urbana (por incluir en ella la ciudad de Neiva, capital del departamento)

La tabla 2 muestra los resultados del tipo de lactancia en el Huila y subregiones. La prevalencia en lactancia fue de $95.8 \%$; LME 58.5 \%; LMP $17.6 \%$; y alimentación complementaria a la LM 19.7 \%; no recibieron LM el 4.1 \%. En la categorización por grupo etario (bimensual) se encuentra que entre mayor es la edad del lactante menor es el porcentaje de LME en su grupo correspondiente, así: en lactantes de 0-1 mes el porcentaje de LME fue 74.8 $\%$; en los de 2-3 meses de $56.4 \%$ y en los de $4-5$ meses de $31.3 \%$. La prevalencia de LME en el Huila en los que viven en área rural fue de $63.8 \%$, y en los que viven en área urbana fue $53.7 \%$.

Por subregiones del departamento, la prevalencia de LME igualmente disminuye con el aumento de la edad de los lactantes. Por orden decreciente, las regiones con mayor prevalencia de LME en <6 meses son: Occidente (68.3\%), Sur (65.9\%), Centro (57.6\%) y Norte (41.3\%). En el grupo etario de 4-5 meses, el orden decreciente es Occidente (46.2\%), Sur (30.8\%), Centro $(28.6 \%)$ y Norte (17.4\%). La prevalencia de LME en área rural fue $70.2 \%$, $64.7 \%, 58.3 \%$ y $57.1 \%$ en zona Occidente, Sur, Centro y Norte, respectivamente. La prevalencia de LME en área urbana fue de $67.8 \%, 65.7 \%, 53.7 \%$ y $37.9 \%$ en Occidente, Norte, Sur y Centro, correspondientemente.

Las tablas 3, 4 y 5 registran los resultados de las respuestas de las madres a preguntas acerca de factores relacionados con LM y uso del biberón en $<6$ meses. Los resultados se comentan adelante en la discusión.

\section{DISCUSIÓN}

El estudio fue representativo para la obtención de indicadores de LM en el Huila y subregiones en áreas urbana y rural. La principal fortaleza se centra en conocer, por primera vez para Huila y subregiones, indicadores de prevalencia de LM en $<6$ meses y factores relacionados. Como limitación encontramos que el muestreo, en su mayoría fue por conveniencia y en menor grado por muestra aleatoria, porque se incluyeron las madres que se podían ubicar por estar sus números de teléfono y direcciones en los servicios de salud o en instituciones del ICBF. Sin embargo, como se considera que hay cobertura universal en salud (es decir, que todas las madres están cubiertas por organismos de salud), hace de esta situación una limitante menor.

La prevalencia de LM en $<6$ meses en el Huila fue de $95.8 \%$ (Colombia, Ensin-2010: $95 \%$ ). Muy similar a países en los cuales la LM tiene altos indicadores como Perú (7) y Bolivia (8), con 98.1 $\%$ y $98.3 \%$, en orden.

La prevalencia de la LM en bebés de 4-5 meses fue de $91.5 \%$ (Colombia, Ensin-2010: 92.5 \%). Comparando con Perú y Bolivia, encontramos 90.1 y $96.8 \%$, respectivamente. Al revisar las cifras de países desarrollados, encontramos que en España es $24.8 \%$ (9); en Noruega es de 82 \% (9); y en EE. UU., 41.5 \% (10); cifras mucho menores que las que presenta nuestro caso.

La prevalencia de LME en $<6$ meses para Huila fue de $58.5 \%$ (Colombia, Ensin-2010: 42.9\%). Comparando, encontramos prevalencias más altas en Perú (72.3\%) y Bolivia (64 \%); y las más bajas en Honduras (31 \%) y Panamá (22 \%) (11). En países desarrollados, como España (28.4 \%), Australia (15.4 \%) (12), EE. UU. (11.9\%), Reino Unido de Gran Bretaña (1.0\%). El Huila tiene una prevalencia buena comparada con países en vía de desarrollo, y muy buena comparada con países desarrollados. 
Si analizamos la prevalencia de la LME por grupos etarios, encontramos que esta disminuye a medida que suman los meses de los bebés. Es de especial importancia la prevalencia de LME a los 4-5 meses, dado que representa, según la OMS, LME “a los 6 meses de edad”. En ellos se encontró una prevalencia para el Huila de 31.3\% (Colombia, Ensin-2010: 23.9 \%). En Perú y Bolivia son 71.1 y $43.6 \%$, respectivamente. Para países desarrollados no fue posible encontrar referencias confiables.

La prevalencia de LME en área rural del Huila fue de 63.8 $\%$, y en urbana de $53.7 \%$. Tan solo en la zona Norte es mayor la prevalencia de LME en área urbana, por incluir a Neiva, su capital (Colombia, Ensin-2010, LME en área rural: 40.4 \%; en área urbana: $43.9 \%$ ). Al analizar resultados por subregiones encontramos que Occidente y Sur tienen mejores prevalencias de LME, tanto en $<6$ meses como a 4-5 meses y están por encima de registro nacional Ensin-2010. La región Norte se encuentra por debajo del registro nacional. Esta información es valiosa porque permite identificar prioridades sobre futuros planes de intervención en favor de la LME en las subregiones.

Tabla 1. Encuesta Lactancia Materna en $<6$ meses 2015, SecresaludHuila-USCO. Información sociodemográfica de los bebes < 6 meses.

\begin{tabular}{|c|c|c|c|c|c|c|c|c|c|c|c|}
\hline & & \multirow{2}{*}{\multicolumn{2}{|c|}{ Total Huila }} & \multicolumn{8}{|c|}{ Subregiones del Huila } \\
\hline & & & & \multicolumn{2}{|c|}{ Centro } & \multicolumn{2}{|c|}{ Norte } & \multicolumn{2}{|c|}{ Occidente } & \multicolumn{2}{|l|}{ Sur } \\
\hline & & $\mathrm{n}$ & $\%$ & $\mathrm{n}$ & $\%$ & $\mathrm{n}$ & $\%$ & $\mathrm{n}$ & $\%$ & $\mathrm{n}$ & $\%$ \\
\hline \multicolumn{2}{|c|}{ Población total } & 335 & 100 & 85 & 25.3 & 80 & 23.8 & 82 & 24.4 & 88 & 26.2 \\
\hline \multirow{2}{*}{ Genero } & Femenino & 154 & 46 & 36 & 42 & 35 & 44 & 39 & 48 & 44 & 50 \\
\hline & Masculino & 181 & 54 & 49 & 58 & 45 & 56 & 43 & 52 & 44 & 50 \\
\hline \multirow{2}{*}{$\begin{array}{l}\text { Área } \\
\text { residencia }\end{array}$} & Rural & 160 & 47.8 & 48 & 56.4 & 14 & 17.5 & 47 & 57.3 & 51 & 58 \\
\hline & Urbano & 175 & 52.2 & 37 & 43.6 & 66 & 82.5 & 35 & 42.7 & 37 & 42 \\
\hline \multirow{3}{*}{$\begin{array}{l}\text { Edad } \\
\text { (meses) }\end{array}$} & $0-1$ & 151 & 45.1 & 38 & 44.7 & 23 & 28.7 & 38 & 46.3 & 52 & 59.0 \\
\hline & $2-3$ & 101 & 30.1 & 23 & 27.0 & 34 & 42.5 & 18 & 21.9 & 23 & 26.1 \\
\hline & $4-5$ & 83 & 24.8 & 21 & 24.7 & 23 & 28.7 & 26 & 31.7 & 13 & 14.7 \\
\hline \multirow{2}{*}{ Edad en días } & Media & 71.1 & & 73.2 & & 85.6 & & 74.8 & & 52.6 & \\
\hline & $\begin{array}{l}\text { Desviación } \\
\text { estándar }\end{array}$ & 54.5 & & 53.4 & & 48.6 & & 60.1 & & 50.8 & \\
\hline
\end{tabular}

Tabla 2. Encuesta Lactancia Materna en $<6$ meses Huila 2015, SecresaludHuila-USCO. Respuestas de las madres sobre si el bebé en las últimas 24 horas había recibido LM y otros líquidos o alimentos

\begin{tabular}{|c|c|c|c|c|c|}
\hline & & \multicolumn{3}{|c|}{\begin{tabular}{|l|} 
Edad del bebé \\
(Categorización bimensual) \\
$\mathrm{n}(\%)$
\end{tabular}} & \multirow[t]{2}{*}{$\begin{array}{l}\text { Total }<6 \text { meses } \\
\text { n }(\%)\end{array}$} \\
\hline & & $\mid 0-1$ & $2-3$ & $4-5$ & \\
\hline \multirow{6}{*}{$\begin{array}{ll}\text { Tipo } & \text { de } \\
\text { Lactancia. } & \end{array}$} & Sí LM & $148(98.0)$ & $98(97.0)$ & $75(90.3)$ & $321(95.8)$ \\
\hline & $\begin{array}{l}\text { LM Exclusiva } \\
\text { (LME) }\end{array}$ & $113(74.8)$ & $57(56.4)$ & $26(31.3)$ & $196(58.5)$ \\
\hline & $\begin{array}{l}\text { LM } \\
\text { Predominante } \\
\text { (LMP) }\end{array}$ & $16(10.5)$ & $16(15.8)$ & $27(32.5)$ & $59(17.6)$ \\
\hline & $\begin{array}{l}\text { LME + LMP } \\
\text { (LM Completa) }\end{array}$ & $129(85.4)$ & $73(72.2)$ & $53(63.8)$ & $255(76.1)$ \\
\hline & $\begin{array}{l}\text { Alimentacion } \\
\text { Complementaria } \\
\text { a la LM }\end{array}$ & $19(12.5)$ & $25(24.7)$ & $22(26.5)$ & $66(19.7)$ \\
\hline & No LM & $3(1.9)$ & $3(2.9)$ & $8(9.6)$ & $14(4.1)$ \\
\hline \multirow{4}{*}{$\begin{array}{l}\text { Prevalencia LME } \\
\text { por subregiones } \\
(\%)\end{array}$} & Zona Centro & $(65.8)$ & (78.3) & (28.6) & $(57.6)$ \\
\hline & Zona Norte & $(56.5)$ & (47.1) & (17.4) & $(41.3)$ \\
\hline & Zona Occidente & $(86.8)$ & $(61.1)$ & $(46.2)$ & $(68.3)$ \\
\hline & Zona Sur & $(80.8)$ & $(52.2)$ & $(30.8)$ & $(65.9)$ \\
\hline \multirow{5}{*}{$\begin{array}{l}\text { Prevalencia de } \\
\text { LME en área } \\
\text { rural vs. urbana } \\
(\%)\end{array}$} & Zona Centro & & & & $\begin{array}{l}\text { Rural: (58.3) } \\
\text { Urbana: (37.9) }\end{array}$ \\
\hline & Zona Norte & & & & $\begin{array}{l}\text { Rural: (57.1) } \\
\text { Urbana: (65.7) }\end{array}$ \\
\hline & Zona Occidente & & & & $\begin{array}{l}\text { Rural: }(70.2) \\
\text { Urbana: }(67.6)\end{array}$ \\
\hline & Zona Sur & & & & $\begin{array}{l}\text { Rural: (64.7) } \\
\text { Urbana: }(537)\end{array}$ \\
\hline & Total Huila & & & & $\begin{array}{l}\text { Rural: (63.8) } \\
\text { Urbana: (53.7) }\end{array}$ \\
\hline
\end{tabular}


Tabla 3. Encuesta Lactancia Materna en $<6$ meses Huila 2015, SecresaludHuila-USCO. Respuestas de las madres sobre factores relacionados con la Lactancia. Total Huila

\begin{tabular}{|c|c|c|c|c|c|}
\hline & \multicolumn{3}{|c|}{$\begin{array}{l}\text { Respuestas de las madres según } \\
\text { la edad del bebé (meses) } \\
\text { Categorización bimensual. n }\end{array}$} & \multirow[t]{2}{*}{$\begin{array}{l}\text { Total de } \\
\text { respuestas } \\
\text { en el }<\text { de } \\
6 \text { meses. } \\
\mathrm{n}(\%)\end{array}$} \\
\hline & & $0-1$ & $2-3$ & $4-5$ & \\
\hline \multicolumn{2}{|c|}{$\begin{array}{l}\text { Madres que respondieron que Sí recibieron } \\
\text { información o asesoría sobre LM por personal de } \\
\text { salud en } 1^{\circ} \text { o } 2^{\circ} \text { dia de vida del bebé. }\end{array}$} & $128(84.8)$ & $91(90.1)$ & $74(89.2)$ & $293(87.5)$ \\
\hline $\begin{array}{l}\text { ¿Qué personal de } \\
\text { salud en el último } \\
\text { año le ha brindado } \\
\text { asesoría en LM? }\end{array}$ & $\begin{array}{l}\text { Auxiliar enfermería } \\
\text { Enfermera } \\
\text { Medico general } \\
\text { Promotora de salud } \\
\text { Pediatra } \\
\text { Otro } \\
\text { Líder comunitario } \\
\text { No recibio orientación } \\
\text { Ginecobstetra } \\
\text { No recuerda }\end{array}$ & $\begin{array}{l}50 \\
36 \\
25 \\
27 \\
5 \\
8 \\
10 \\
7 \\
4 \\
1\end{array}$ & $\begin{array}{l}44 \\
30 \\
25 \\
16 \\
7 \\
9 \\
4 \\
4 \\
3 \\
0\end{array}$ & $\begin{array}{l}36 \\
37 \\
29 \\
15 \\
11 \\
6 \\
6 \\
7 \\
1 \\
2\end{array}$ & $\begin{array}{l}130(27.9) \\
103(22.1) \\
79(16.9) \\
58(12.4) \\
23(4.9) \\
23(4.9) \\
20(4.3) \\
18(3.8) \\
8(1.7) \\
3(0.6)\end{array}$ \\
\hline $\begin{array}{l}\text { Momento en que el } \\
\text { personal de salud } \\
\text { brindó asesoría en } \\
\text { LM. }\end{array}$ & $\begin{array}{l}\text { Antes del embarazo } \\
\text { En el embarazo } \\
1^{\circ} \text { dia de vida del bebé } \\
2^{\circ} \text { dia de vida del bebe } \\
\text { Consulta postparto } \\
\text { Consulta c y d del bebe } \\
\text { No recibió } \\
\text { No recuerda } \\
\text { Total respuestas }\end{array}$ & $\begin{array}{l}21 \\
91 \\
42 \\
12 \\
10 \\
3 \\
8 \\
1 \\
187\end{array}$ & $\begin{array}{l}14 \\
73 \\
42 \\
10 \\
7 \\
4 \\
4 \\
0 \\
157\end{array}$ & $\begin{array}{l}14 \\
76 \\
31 \\
9 \\
15 \\
6 \\
10 \\
0 \\
158\end{array}$ & $\begin{array}{l}49(9.7) \\
240(47.7) \\
115(22.8) \\
31(6.1) \\
32(6.3) \\
13(2.5) \\
22(4.3) \\
1(0.1) \\
503(100)\end{array}$ \\
\hline $\begin{array}{l}\text { ¿Qué liquido o } \\
\text { alimento recibió el } \\
\text { bebé inmediatamente } \\
\text { después de nacer y } \\
\text { antes de ser } \\
\text { amamantado? }\end{array}$ & $\begin{array}{l}\text { Leche de formula } \\
\text { Agua } \\
\text { Dextrosa } \\
\text { No recuerda/no sabe } \\
\text { No recibia liquidos } \\
\text { Total de respuestas }\end{array}$ & $\begin{array}{l}15 \\
0 \\
3 \\
6 \\
68 \\
98\end{array}$ & $\begin{array}{l}12 \\
1 \\
0 \\
3 \\
62 \\
78\end{array}$ & $\begin{array}{l}19 \\
4 \\
1 \\
10 \\
43 \\
77\end{array}$ & $\begin{array}{l}46(18.6) \\
5(2.0) \\
4(1.6) \\
19(7.6) \\
173(70.0) \\
247(100)\end{array}$ \\
\hline $\begin{array}{l}\text { En qué momento el } \\
\text { bebé empezó a } \\
\text { recibir LM después } \\
\text { de nacer? }\end{array}$ & $\begin{array}{l}1^{\circ} \text { hora de nacido } \\
\text { Luego } 1 \text { hora de nacer } \\
\text { Después del } 1 \text { día } \\
\text { Nunca recibió L M } \\
\text { No recuerda/no sabe } \\
\text { Total de respuestas }\end{array}$ & $\begin{array}{l}109 \\
33 \\
6 \\
3 \\
0 \\
151\end{array}$ & $\begin{array}{l}63 \\
28 \\
8 \\
2 \\
0 \\
101 \\
\end{array}$ & $\begin{array}{l}60 \\
15 \\
7 \\
1 \\
0 \\
83\end{array}$ & \begin{tabular}{|l|}
$232(66.3)$ \\
$76(22.7)$ \\
$21(6.3)$ \\
$6(1.8)$ \\
0 \\
$335(100)$ \\
\end{tabular} \\
\hline $\begin{array}{l}\text { Razones por las } \\
\text { cuales los bebés } \\
\text { nunca recibieron } \\
\text { leche materna. } \\
\text { ¿Qué motivo } \\
\text { tuvieron para que el } \\
\text { bebé no recibiera } \\
\text { más leche materna } \\
\text { (suspensión de la } \\
\text { leche materna)? }\end{array}$ & $\begin{array}{l}\text { Mamá enferma/débil } \\
\text { No le bajó leche } \\
\text { Recién nacido enfermo } \\
\text { Otra razón } \\
\text { Total de respuestas } \\
\text { Mamá enferma/débil } \\
\text { Problema del bebé para } \\
\text { tomar seno } \\
\text { Lesión del pezón } \\
\text { Se le secó la leche } \\
\text { Mamá necesitó trabajar } \\
\text { Mastitis } \\
\text { Otra } \\
\text { Total de respuestas }\end{array}$ & $\begin{array}{l}2 \\
2 \\
1 \\
0 \\
5 \\
0 \\
\\
0 \\
0 \\
1 \\
\\
0 \\
0 \\
0 \\
1\end{array}$ & $\begin{array}{l}0 \\
0 \\
1 \\
1 \\
2 \\
0 \\
1 \\
1 \\
0 \\
0 \\
1 \\
1 \\
0 \\
3 \\
\end{array}$ & $\begin{array}{l}0 \\
0 \\
0 \\
0 \\
0 \\
1 \\
5 \\
4 \\
3 \\
1 \\
0 \\
0 \\
14\end{array}$ & $\begin{array}{l}2(28.5) \\
2(28.5) \\
2(28.5) \\
1(14.2) \\
7(100) \\
1(5.5) \\
6(33.3) \\
4(22.2) \\
4(22.2) \\
2(11.1) \\
1(5.5) \\
0 \\
18(100)\end{array}$ \\
\hline $\begin{array}{l}\text { ¿Qué otro alimento, } \\
\text { aparte de LM, recibió } \\
\text { el bebé en las últimas } \\
24 \text { horas? }\end{array}$ & $\begin{array}{l}\text { Agua } \\
\text { Leche de fórmula } \\
\text { Sopa } \\
\text { Jugo de fruta sin leche } \\
\text { Colada } \\
\text { Compota } \\
\text { Alimentos licuados } \\
\text { Pollo } \\
\text { Fruta_entera/trozos) } \\
\text { Carne de res_(trozos) } \\
\text { Agua de panela } \\
\text { Cereal } \\
\text { Papa (trozos) } \\
\text { Arroz } \\
\text { Puré } \\
\text { Frijol, arveja,_lenteja } \\
\text { Agua de azúcar } \\
\text { Total de respuestas }\end{array}$ & $\begin{array}{l}23 \\
16 \\
2 \\
2 \\
2 \\
1 \\
1 \\
0 \\
0 \\
0 \\
2 \\
0 \\
1 \\
1 \\
0 \\
0 \\
0 \\
53\end{array}$ & $\begin{array}{l}32 \\
22 \\
5 \\
4 \\
3 \\
2 \\
0 \\
1 \\
2 \\
1 \\
0 \\
1 \\
1 \\
1 \\
1 \\
1 \\
0 \\
81\end{array}$ & $\begin{array}{l}70 \\
36 \\
17 \\
13 \\
11 \\
9 \\
7 \\
6 \\
4 \\
4 \\
3 \\
4 \\
3 \\
4 \\
2 \\
2 \\
2 \\
207\end{array}$ & $\begin{array}{l}125(36.6) \\
74(21.7) \\
24(7.0) \\
19(5.5) \\
16(4.6) \\
12(3.5) \\
8(2.3) \\
7(2.0) \\
6(1.7) \\
5(1.4) \\
5(1.4) \\
5(1.4) \\
5(1.4) \\
6(1.7) \\
3(0.8) \\
3(0.8) \\
2(0.5) \\
341(100)\end{array}$ \\
\hline
\end{tabular}


La prevalencia de la LMP en el Huila fue de $17.6 \%$ (Colombia, Ensin-2010: $10.3 \%$ ).

La LME sumada a la LMP (interpretada como lactancia materna completa), es decir, que los lactantes tomen exclusivamente leche materna o leche materna y líquidos claros no lácteos, es de 76.1 \% (Colombia, Ensin-2010: $53.1 \%$ ).

Las madres recibieron orientación por personal de salud sobre LM en el primer o segundo día de vida del bebé en 87.5 \% de los casos (Colombia, Ensin-2010: 70.4 \%; y Huila: $79.2 \%$ ).

Las madres respondieron que el personal que brindó orientación sobre LM en el último año tenía por profesión o tarea: auxiliar de enfermería (27.9\%), enfermera (22.1\%), médico general (16.9\%), promotora de salud (12.4\%) y pediatra (4.9\%). El $4.2 \%$ de las madres respondió que no había recibido ninguna instrucción u orientación. La Ensin-2010 reportó que no han recibido orientación sobre LM en los últimos cinco años en Colombia el $50.3 \%$ de la población, y en el Huila el $41.7 \%$.

En las respuestas recogidas sobre el momento en que el personal de salud, en el último año, brindó orientación sobre
LM se encontró que, en orden decreciente es: $47.7 \%$ mientras estaba embarazada; el 22.8 \% en el primer día en que nació el bebé; el $9.7 \%$ antes del embarazo; el $6.3 \%$ en la consulta del post-parto; el $2.5 \%$ en la consulta de crecimiento y desarrollo del bebé. El $4.3 \%$ de las respuestas fue que no habían recibido orientación. En la Ensin-2010 no se reporta este tipo de información. Llama la atención que las madres dicen que muy pocas veces reciben orientación en el momento de la consulta del post-parto o en la consulta de crecimiento y desarrollo del bebé. Estos corresponden con momentos muy propicios para el fomento de la LME o para la solución de problemas relacionados con la lactancia.

El líquido o alimento más frecuente utilizado en el recién nacido, antes de recibir LM, fue: fórmula en $18.6 \%$, agua $2.0 \%$ y dextrosa $1.6 \%$. En un $70 \%$ de las respuestas no se ofreció líquido o alimento al bebé. De la muestra total, el $50 \%$ de los bebés no recibió liquido o alimento antes de ser amamantados (Ensin-2010 no reporta).

Tabla 4. Encuesta Lactancia Materna en $<6$ meses, Huila 2015, SecresaludHuila-USCO. Respuestas de las madres sobre el momento de inicio del biberón

\begin{tabular}{|l|l|l|l|}
\hline $\begin{array}{l}\text { Edad de inicio del biberon } \\
\text { (días) }\end{array}$ & $\begin{array}{l}\text { Frecuencia, de los bebes }<6 \\
\text { meses, que usaron biberón } \\
\text { el día anterior }\end{array}$ & $\begin{array}{l}\text { Porcentaje de bebes que } \\
\text { usaron biberón (n=107) } \\
\text { según el momento de inicio } \\
\text { del biberón. }\end{array}$ & $\begin{array}{l}\text { Porcentaje de bebés } \\
\text { que usaron biberón } \\
\text { del día anterior con } \\
\text { respecto al total de } \\
\text { población <6 meses } \\
\text { (n=335). }\end{array}$ \\
\hline $0-29$ & 52 & 48.6 & 15.5 \\
\hline $30-59$ & 8 & 7.5 & 2.4 \\
\hline $60-89$ & 7 & 8.4 & 2.7 \\
\hline $90-119$ & 7 & 6.5 & 2.1 \\
\hline $120-149$ & 1 & 6.5 & 2.1 \\
\cline { 1 - 1 } $150-179$ & 23 & 0.9 & 0.3 \\
\cline { 1 - 1 } $\begin{array}{l}\text { Sin dato de edad de inicio } \\
\text { del biberón. }\end{array}$ & 23 & 21.5 & 6.9 \\
\hline $\begin{array}{l}\text { Total de los que usaron } \\
\text { biberón }\end{array}$ & 107 & 100 & 31.9 \\
\hline
\end{tabular}

Tabla 5. Encuesta Lactancia Materna en $<6$ meses, Huila 2015, SecresaludHuila-USCO. Respuestas de las madres sobre el momento de inicio del biberón en lactantes de 4-5 meses

\begin{tabular}{|c|c|c|c|}
\hline $\begin{array}{l}\text { Edad de inicio del } \\
\text { biberón (días) }\end{array}$ & $\begin{array}{l}\text { Frecuencia de los que } \\
\text { usaron biberón el día } \\
\text { anterior. }\end{array}$ & $\begin{array}{l}\text { Porccntajc de bebes de } \\
4-5 \text { meses que usaron } \\
\text { bibcrón el día anterior } \\
(\mathrm{n}=35) \text { según el } \\
\text { momento de inicio del } \\
\text { bibcrón. }\end{array}$ & $\begin{array}{l}\text { Porccntaje de bebćs de } \\
4-5 \text { meses que usaron } \\
\text { bibcrón cl día anterior } \\
\text { con respecto al total de } \\
\text { bebes de } 4-5 \text { meses } \\
(n=83)\end{array}$ \\
\hline $0-29$ & 18 & 51.4 & 21.7 \\
\hline $30-59$ & 2 & 5.7 & 2.4 \\
\hline $60-89$ & 3 & 8.6 & 3.6 \\
\hline $90-119$ & 6 & 17.1 & 7.2 \\
\hline $120-149$ & 5 & 14.3 & 6.0 \\
\hline $150-179$ & 1 & 2.9 & 1.2 \\
\hline Total & 35 & 100 & 42.2 \\
\hline
\end{tabular}


Tabla 6. Indicadores de Lactancia Materna y Lactancia Materna Exclusiva en $<6$ meses en países desarrollados y en via de desarrollo

\begin{tabular}{|c|c|c|c|c|c|c|}
\hline Países & \begin{tabular}{lr}
\multicolumn{3}{l}{ Prevalencia } \\
inicio de & LM \\
\multicolumn{3}{l}{ en la primera } \\
hora \\
nacido de \\
(bebés \\
meses) \\
$\%$
\end{tabular} & $\begin{array}{l}\text { Prevalencia } \\
\text { de: Haber } \\
\text { recibido } \\
\text { alguna vez } \\
\text { LM (bebés } \\
<6 \text { meses). } \\
\%\end{array}$ & $\begin{array}{l}\text { Prevalencia de } \\
\text { LM a los } 6 \\
\text { meses (a los } 4- \\
5 \text { meses de } \\
\text { edad). } \\
\%\end{array}$ & $\begin{array}{l}\text { Prevalencia } \\
\text { de LM en } \\
<6 \text { meses. } \\
\%\end{array}$ & $\begin{array}{l}\text { Prevalencia LM } \\
\text { exclusiva en } \\
\text { bcbćs de } 4-5 \\
\text { meses. ("LME a } \\
\text { los } 6 \text { meses"). } \\
\%\end{array}$ & $\begin{array}{l}\text { Prevalencia } \\
\text { lactancia } \\
\text { materna } \\
\text { exclusiva } \\
\text { (LME) en }<6 \\
\text { meses. } \\
\%\end{array}$ \\
\hline Perú* & 55.6 & 98.6 & 90.1 & 98.1 & 71.1 & 72.3 \\
\hline Bolivia $\uparrow$ & 78 & 97.7 & 96.8 & 98.3 & 43.6 & 64 \\
\hline $\begin{array}{l}\text { Huila (presente } \\
\text { estudio) }\end{array}$ & 69.3 & 98.2 & 91.5 & 95.8 & 31.3 & 58.5 \\
\hline Guatemala: & 56 & & & & & 53 \\
\hline El Salvador: & 42 & & & & & 47 \\
\hline Colombia** & 57 & 96 & 92.5 & 95 & 23.9 & 43 \\
\hline Ecuador & 55 & & & & & 40 \\
\hline Haitit & 47 & & & & & 40 \\
\hline Brasil : & 43 & & & & & 39 \\
\hline Argentinat: & 53 & & & & & 33 \\
\hline Costa Ricat & 60 & & & & & 33 \\
\hline Cubat & 48 & & & & & 33 \\
\hline Nicaraguał & 68 & & & & & 32 \\
\hline Honduras: & 64 & & & & & 31 \\
\hline Panamát & 47 & & & & & 22 \\
\hline Noruegatit & & & 82 & & & \\
\hline Españaṭ & & & 24.8 & & & 28.4 \\
\hline Australiatt & & $\begin{array}{l}90.2 \\
(<1 \mathrm{mes})\end{array}$ & & & & 15.4 \\
\hline $\begin{array}{l}\text { Estados Unidos } \\
\text { de América }\end{array}$ & & 73.8 & 41.5 & & & 11.9 \\
\hline $\begin{array}{ll}\text { Reino } & \text { Unido } \S \\
(2009) & \end{array}$ & & & & & & 1 \\
\hline \multicolumn{7}{|c|}{ 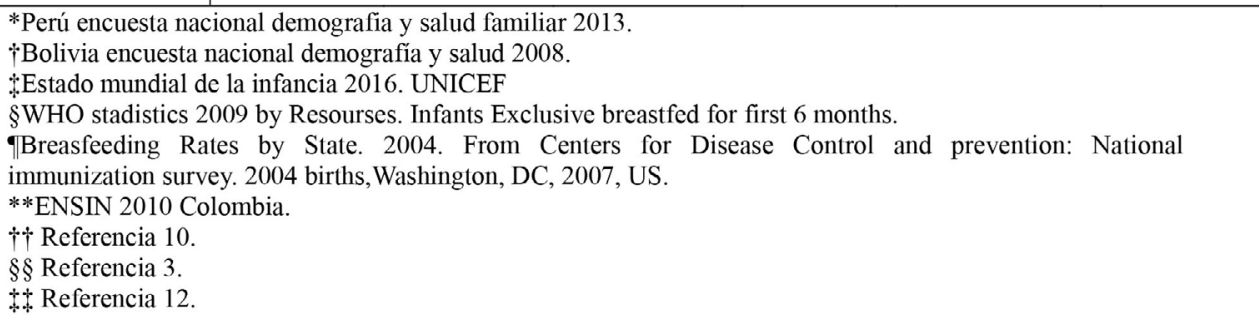 } \\
\hline
\end{tabular}

Las respuestas de las madres sobre el momento en que el bebé empezó a recibir LM luego de su nacimiento fueron: 69.3 $\%$ dentro de primera hora de nacer; 22.7 \% luego de la primera hora de nacido, pero durante el primer día; y el $6.3 \%$ después del primer día de nacido. El $1.8 \%$ de los bebés nunca recibió LM (Ensin-2010 no reporta).

Las razones por la cuales los bebés (6 bebés, es decir, $1.8 \%$ ) nunca recibieron LM fueron (en total hubo 7 respuestas): 2 razones señalaron que la madre estaba muy enferma (28.5 \%); 2 indicaron que no le bajó leche a la madre (28.5\%); 2 dijeron que el bebé estaba enfermo (28.5 \%); y 1 razón no fue referida (Ensin-2010 no reporta).

Entre las razones por las cuales el bebé dejó de recibir LM, se registró que el 4.1 \% de madres respondieron que sus bebés no recibieron LM el día anterior. Los motivos son referidos como: problemas del bebé para agarrar el seno (33.3\%), lesiones o enfermedades en el pezón o el seno (22.2 \%), se le secó la leche a la madre (22.2\%); necesidad de que la madre trabaje (11.1\%).

En cuanto el uso del biberón en $<6$ meses, se encontró que el 31.9 \% de bebés usaron biberón el día anterior de la encuesta (en Colombia, Ensin-2010: 44.5 \%). Entre los que sí usaron biberón, la mayoría (48.6 \%) lo inició entre su nacimiento y los 29 días de edad. En el grupo de bebés de 4-5 meses de edad, el 42.2 \% usó biberón (en Colombia, Ensin-2010: 55.2 \%). De los lactantes de 4-5 meses que usaron biberón, el 51.4 \% inició su uso dentro de los 29 días posteriores al nacimiento.

Con respecto a los líquidos o alimentos que recibió el bebé que es lactado, en las 24 horas previas las madres respondieron: agua (36.6\%), formula láctea (21.7\%), sopa (7 \%), jugo de 
fruta (5.5\%), colada (4.6\%), compota (3.5\%), alimentos licuados (2.3\%) y otros. Según Ensin-2010 Colombia, el $33.2 \%$ de los $<6$ meses encuestados, que son amamantados, recibieron formula artificial el día anterior (Huila, Ensin-2010: $23.0 \%$ ).

\section{CONCLUSIONES}

El estudio en general aportó conocimiento valioso acerca de las prevalencias y factores relacionados con la LM en el Huila. Podemos destacar las siguientes conclusiones:

El tamaño de la muestra y la metodología desarrollada generaron resultados de confiabilidad y representatividad para el Huila y subregiones, tanto en área rural como urbana.

En $<6$ meses, en el Huila (y sus subregiones) era desconocida la prevalencia de la LM, LME, LMP y LMC. Las prevalencias encontradas estuvieron por encima de las medias para Colombia, Ensin-2010.

Se estableció un orden de las regiones, de mayor a menor prevalencia de LME en $<6$ meses, así: zona Occidente, Sur, Centro y Norte.

Se encontraron factores de interés relacionados con LM que podrán servir para mejorar la comprensión de esta práctica en nuestra población.

Se sugiere que la encuesta LM en $<6$ meses en el Huila sea realizada cada cinco años para evaluar la tendencia de indicadores y medir así el impacto de los planes de intervención.

\section{R E F E R E N C I A S}

1. Ruth A. Lawrence \& Robert M. Lawrence. The revolution in infant feeding. In Breastfeeding: A guide for the medical profession. 8. ${ }^{a}$ ed. Elsevier; 2016. 1-33p.
2. OMS, OPS. La alimentación del lactante y el niño pequeño. Washington, D.C.: c2010.

3. Victora CG, Bahl R, Barros AJ, França GV, Horton S, Krasevec J, Murch S, et al. Breastfeeing in the 21st century: epidemiology, mechanisms, and lifelong effect. Lancet: 2016;387(10017):47590.

4. ICBF, PROFAMILIA, INS, Escuela de Nutrición y Dietética. Encuesta Nacional de la Situación nutricional en Colombia, 2005. 1. ${ }^{\text {a }}$ ed. Bogotá: 2006.

5. PROFAMILIA, ICBF, INS, Ministerio de Salud y Protección Social de Colombia. Encuesta nacional de situación nutricional Colombia 2010. Bogotá: ENSIN; agosto 2011.

6. OMS, UNICEF. Indicadores para evaluar prácticas de alimentación del lactante y niño pequeño. Parte 1, Definiciones. Conclusiones de reunión de consenso; 2007 nov. 6-8;Washington, D.C (EE.UU.).

7. Instituto Nacional de Estadística e Informática Perú. Encuesta demográfica y salud familiar. Sección 10: Lactancia y nutrición de niños, niñas y madres. ENDES; 2013.

8. Instituto Nacional de Estadística Bolivia. Ministerio de Salud y deportes. Encuesta nacional de demografía y salud. Bolivia: 2008.

9. Moran Barrio MA. Comparación de la situación de lactancia materna entre España y Noruega [trabajo de grado en Enfermería;digital]. [Valladolid]: Universidad de Valladolid; 2013. Disponible en: https://uvadoc.uva.es/ bitstream/10324/5026/1/TFG-M40.pdf

10. National Inmunization Survey's Landine Sampling frame. 2013. USA

11. UNICEF. Estado Mundial de la Infancia 2016. Una oportunidad para cada niño. Tablas estadísticasTabla 2: Nutrición página 122. Junio 2016.

12. Australian Government. Australian Institute of Health and Welfare. Australian National Infant feeding Survey 2010. Indicator results. Disponible en: www.aihw.gov.au/ downloadasset.aspx?id=10737420925 